\title{
QUALIDADE FISIOLÓGICA, SANITÁRIA E ANÁLISE DE ISOENZIMAS DE SEMENTES DE AVEIA-PRETA TRATADAS COM DIFERENTES FUNGICIDAS ${ }^{1}$
}

\author{
FERNANDO AUGUSTO HENNING²; LILIANE MARCIA MERTZ2; PAULO DEJALMA ZIMMER; \\ MARCUS DAVI FERREIRA TEPLIZKY²
}

\begin{abstract}
RESUMO - A aveia-preta é uma gramínea bastante rústica empregada como forragem e adubação verde de inverno em sistemas de rotação de cultura. A baixa qualidade fitossanitária das sementes de aveia-preta devido ao manejo inadequado na fase de colheita e pós-colheita e a oferta irregular de sementes básicas constituem-se em um dos fatores limitantes à produção. O objetivo neste trabalho foi avaliar o desempenho de um Bioprotetor (Trichoderma spp.) e dos fungicidas químicos (Carbendazin + Thiram e Carboxin + Thiram) na qualidade fisiológica, sanitária e na atividade enzimática de sementes de aveia-preta. Após o tratamento das sementes, avaliou-se a qualidade fisiológica pelos testes de germinação e vigor (primeira contagem da germinação e índice de velocidade de emergência). Para a análise sanitária, foi utilizado o método do papel-filtro (blotter test). As atividades das enzimas álcool desidrogenase, fosfatase ácida e esterase foram avaliadas por meio da técnica de eletroforese. Por meio dos testes de vigor e germinação foi observado melhor desempenho das sementes tratadas com fungicidas químicos. Nos resultados da análise sanitária observou-se a presença dos fungos Bipolaris sp. e Fusarium spp., sendo que, o tratamento com Bioprotetor não diferiu estatisticamente da testemunha, apresentando maior incidência de fungos quando comparado aos tratamentos com fungicidas químicos. Em relação às alterações bioquímicas, nas sementes não tratadas e nas tratadas com Bioprotetor, é observado incremento na atividade enzimática das enzimas avaliadas de acordo com abstract indicado degenerativas decorrentes da ação de fungos.
\end{abstract}

Termos para indexação: Avena strigosa, eletroforese, qualidade sanitária, bioprotetor.

\section{PHYSIOLOGICAL, SANITARY QUALITY AND ISOENZYME ANALYSIS OF BLACK OAT SEEDS TREATED WITH DIFFERENT FUNGICIDES}

\begin{abstract}
Black-oat is a rustic grass used as livestock fodder as well as winter green fertilization in crop rotation systems. Low sanitary quality of black oat seeds due to inadequate handling at harvest and in after-harvest phase is one of the limiting factors to production. The objective of this study was to evaluate the performance of a bioprotetor (Trichoderma spp.) and chemical fungicides (Carbendazin + Thiram and Carboxin + Thiram) in the physiological and sanitary quality of black oat seeds and in their enzymatic activities. After the treatment the physiological quality of the seeds was evaluated by germination and vigor tests (speed of emergence and first germination count). The sanitary analysis was carried out by the blotter test. Alcohol desidrogenase, acid fosfatase and esterase activity were evaluated by electrophoresis. Vigour and germination tests showed that seeds
\end{abstract}

${ }^{1}$ Submetido em 15/03/2007. Aceito para publicação em 15/01/2008.

${ }^{2}$ Eng. Agr., Discentes do Programa de Pós-Graduação em C\&T de Sementes

- FAEM/UFPel. e-mail: fernandohenning@yahoo.com.br, Campus
Universitário, 354, Cep: 96010-900, Pelotas, RS, Brasil.

${ }^{3}$ Eng. Ag., Dr. Prof. do Departamento de Fitotecnia/FAEM/ UFPel., e-mail: djzimmer@ufpel.edu.br 
treated with chemical fungicides performed better. In the sanitary analysis, the presence of fungi Bipolaris sorokiniana and Fusarium graminearum was observed. The treatment with bioprotetor did not differ statistically from the control, showing higher fungi incidence when compared to the seeds treated with chemical fungicides. In relation to biochemical alterations, the treated seeds and the ones treated with bioprotetor presented an increase in the isoenzymatic activity in all the evaluated enzymes indicating degenerative transformations due to fungi action.

Index terms: Avena strigosa, electrophoresis, seed treatment, bioprotetor.

\section{INTRODUÇÃO}

A estacionalidade na produção de forragem, devido ao crestamento da pastagem nativa provocada pelas freqüentes geadas, leva a insuficiência na produção de forragem em quantidade e qualidade. Neste sentido, a aveia-preta (Avena strigosa Schreb.), juntamente com o azevém-anual (Lolium multiflorum Lam.), são as espécies forrageiras de inverno mais cultivadas e de maior importância econômica no Estado do Rio Grande do Sul (Farias et al.; 2002).

Um dos problemas que envolvem a formação de pastagens no Brasil é a variação apresentada na qualidade das sementes de espécies forrageiras existentes no comércio. A oferta irregular de sementes básicas resulta na comercialização de sementes de baixa qualidade física, fisiológica, genética e sanitária, com reflexos negativos na implantação da cultura e no aumento dos custos produção (Lucca Filho et al., 1999).

Em trabalho desenvolvido por Belmonte e LuccaFilho (2002), avaliando a qualidade das sementes de aveia-preta produzidas no estado do RS, observou-se que aproximadamente $20 \%$ das sementes analisadas, não atenderam ao padrão mínimo de germinação exigido para a comercialização.

A qualidade sanitária também influencia o estabelecimento de plantas no campo. Segundo Neergard (1979), diversos fungos quando presentes nas sementes podem provocar aborto, deformações, podridões, descolorações e necroses, com reflexos na diminuição da viabilidade e no vigor das mesmas. Em estudos conduzidos por Bevilaqua e Pierobom (1995), foram detectados fungos patogênicos, de armazenamento e outros microrganismos associados às sementes de aveia-preta, sendo que, a alta incidência de patógenos importantes como os causadores de helmintosporiose tiveram influência direta na germinação das sementes e no estabelecimento das plantas.

Além dos efeitos observados no desempenho fisiológico das sementes, a infecção por microrganismos pode promover também, alterações nos padrões de certas enzimas, o que pode ser atribuído ao próprio processo de deterioração da semente. Segundo Delouche e Baskin (1973), a atividade enzimática pode indicar transformações degenerativas nas sementes. Em trabalho conduzido por Silva et al. (2000) a infecção das sementes com Aspergillus flavus promoveu alterações tanto na intensidade como no número de bandas dos padrões isoenzimáticos da álcool-desidrogenase e malato-desidrogenase.

O tratamento de sementes é uma alternativa simples e econômica, que garante maiores percentuais de germinação e emergência das plântulas, isto é resultante do retardamento da infecção primária das sementes e, redução de inóculo de patógenos radiculares e de solo (Balardin e Loch, 1987). Atualmente além dos fungicidas químicos, encontram-se disponíveis no mercado biopropetores, os quais surgiram no intuito de reduzir a utilização de pesticidas sintéticos, os riscos aos operadores e os possíveis prejuízos ao meio ambiente.

Dentre estes fungicidas biológicos, alguns são formulados com isolados de Trichoderma spp., pois estes apresentam amplitude de ação no antagonismo a fungos e bactérias. Esses microrganismos são atóxicos ao homem e aos animais. Além dos efeitos de Trichoderma spp. no controle de patógenos de plantas, certas linhagens podem ter efeito estimulatório no crescimento e no florescimento de plantas hortícolas (Baker, 1989). A adição de Trichoderma spp. a solos autoclavados aumentou a emergência e a matéria seca de plântulas de tomate e de fumo (Windham et al., 1986). Em solos naturais, observou-se que isolados de Trichoderma spp. proporcionaram maior germinação de sementes, emergência e vigor de plântulas de berinjela (Martin-Corder e Melo, 1998). Sementes de algodão submetidas aos tratamentos com T. harzianum, carboxin+thiram e carbendazin+thiram apresentaram porcentagem de germinação estatisticamente superior à testemunha (Faria et al., 2002).

Diante do exposto, o objetivo neste trabalho foi avaliar a qualidade fisiológica, sanitária e a atividade enzimática de 
sementes de aveia-preta tratadas com diferentes fungicidas.

\section{MATERIAL E MÉTODOS}

O trabalho foi realizado no Laboratório Didático de Análise de Sementes do Departamento de Fitotecnia, UFPel/ FAEM. Foram utilizadas sementes de aveia-preta produzidas na safra 2005/06, no município de Capão do Leão - RS.

As sementes foram tratadas em sacos plásticos utilizandose os seguintes fungicidas: biopropetor (Trichoderma spp.) na dose de $150 \mathrm{~mL}$ para cada $100 \mathrm{Kg}$ de sementes, fungicida químico (Carbendazin + Thiram) na dose de $200 \mathrm{~mL}$ para cada $100 \mathrm{~kg}$ de sementes, fungicida químico (Carboxin + Thiram) na dose de $250 \mathrm{~mL}$ para cada $100 \mathrm{~kg}$ de sementes. Foi utilizado ainda um tratamento testemunha sem tratamento fungicida.

Após o tratamento das sementes, realizou-se a avaliação da qualidade fisiológica, sanitária e da atividade das isoenzimas fosfatase ácida, esterase e álcool desidrogenase.

A qualidade fisiológica das sementes foi avaliada por meio dos testes de germinação e vigor (Primeira contagem da germinação e Índice de velocidade de emergência).

No teste de germinação as sementes foram semeadas em rolos de papel toalha (Germitest) umedecidos e colocados em temperatura constante $\left(25^{\circ} \mathrm{C}\right)$; utilizaram-se três repetições de 200 sementes (50 sementes por rolo). A avaliação foi realizada após o quinto dia da semeadura de acordo com os critérios das Regras para análise de sementes (Brasil, 1992).

Para o teste de primeira contagem da germinação, foram utilizados os dados de plântulas normais obtidas na primeira contagem do teste de germinação (Krzyzanowski et al., 1999). $\mathrm{Na}$ determinação do Índice de Velocidade de Emergência (IVE) as sementes foram semeadas em solo no interior de bandejas plásticas, permanecendo em local com temperatura controlada $\left(25^{\circ} \mathrm{C}\right)$. Foram utilizadas quatro repetições de 100 sementes. A contagem foi iniciada no momento em que a primeira plântula emergiu e foi finalizada quando o número de plântulas permaneceu estável por mais de três dias. Em seguida calculou-se o índice de velocidade de emergência conforme descrito por Krzyzanowski et al. (1999).

A qualidade sanitária das sementes foi avaliada por meio do teste do papel filtro (Neergaard, 1979). Foram utilizadas quatro repetições de 100 sementes, colocadas sobre três folhas de papel filtro em caixas plásticas, tipo gerbox, sendo utilizadas 25 sementes por caixa. Em seguida as amostras foram incubadas a temperatura de $20 \pm 2^{\circ} \mathrm{C}$, por período de sete dias e regime luminoso de 12 horas de luz. Finalizado esse período, as sementes foram examinadas individualmente com auxílio de microscópio estereoscópio e aumento de até 60x. Quando necessário, foram realizadas preparações de lâminas microscópicas para a identificação dos fungos.

As avaliações das atividades das enzimas esterase, fosfatase ácida e álcool desidrogenase foram realizadas utilizando-se o sistema de eletroforese vertical em gel de poliacrilamida. O material vegetal foi composto de 10 plântulas com cinco dias, as quais foram maceradas em mortar de porcelana sobre cubos de gelo. Aproximadamente $200 \mathrm{mg}$ de cada extrato vegetal foram colocados em tubo de microcentrífuga acrescidos de solução extratora composta pelo tampão do gel (Borato de Lítio 0,2M a pH 8,3 + Tris Citrato $+0,2 \mathrm{M}$ à pH 8,3$)+0,15 \%$ de 2 -mercaptoetanol) na proporção $1: 2(\mathrm{p} / \mathrm{v})$. Realizou-se a eletroforese em géis de poliacrilamida $7 \%$, aplicando-se $20 \mu \mathrm{L}$ de cada amostra. Foram utilizados os sistemas de coloração descritos por Scandalios (1969) e Alfenas (1998). A interpretação dos resultados foi baseada na análise visual dos géis de eletroforese, levandose em consideração a presença e ausência, e a intensidade de expressão de cada uma das bandas.

Para análise estatística dos dados obtidos no experimento, realizou-se à análise de variância, com delineamento inteiramente casualizado. A comparação entre as médias dos genótipos foi efetuada pelo teste de Duncan, a $5 \%$ de probabilidade utilizando o software SASM-AGRI (Canteri et al., 2001).

\section{RESULTADOS E DISCUSSÃO}

Pelos dados de germinação, primeira contagem e IVE foram observados que sementes de aveia-preta tratadas com fungicidas químicos, apresentam qualidade fisiológica superior em relação às sementes tratadas com biopropetor biológico e a testemunha sem tratamento (Tabela 1). Esse mesmo resultado foi observado por Mertz et al. (2009), em trabalho com sementes de soja.

Dos produtos químicos utilizados no trabalho, apenas o carboxin + thiram é recomendado para o tratamento de sementes de aveia-preta, mas de acordo com os resultados obtidos, o carbendazin + thiram pode ser uma alternativa no tratamento de sementes dessa cultura, pois dentre todos os tratamentos foi o que apresentou melhores resultados. Em trabalho desenvolvido por Henning et al. (2004), sementes de soja tratadas com carbendazin + thiram apresentaram qualidade fisiológica superior quando comparada a observada em tratamentos com outros fungicidas. 
TABELA 1. Qualidade Fisiológica de sementes de aveia-preta, produzidas no município do Capão do Leão, safra 2005/06, tratadas com diferentes fungicidas.

\begin{tabular}{cccc}
\hline Tratamento & Germinação $(\%)$ & Primeira Contagem $(\%)$ & IVE $(\%)$ \\
\hline Testemunha & $75 \mathrm{~b}$ & $64 \mathrm{~b}$ & $19,8 \mathrm{c}$ \\
Carbendazin + Thiram & $85 \mathrm{a}$ & $78 \mathrm{a}$ & $33,6 \mathrm{a}$ \\
Carboxin + Thiram & $82 \mathrm{a}$ & $75 \mathrm{a}$ & $30,4 \mathrm{ab}$ \\
Trichoderma spp. & $80 \mathrm{ab}$ & $72 \mathrm{ab}$ & $28,8 \mathrm{bc}$ \\
\hline
\end{tabular}

Comparação de médias feita pelo teste de Duncan, a 5\% de probabilidade.

Quanto aos dados de incidência de fungos, foram encontrados Fusarium spp. e Bipolaris sp., os quais são patógenos rotineiramente associados a sementes de aveia-preta (Tabela 2). Esses resultados corroboram com os estudos realizados por Farias et al. (2002), nos quais se verificou que sementes de aveia-preta produzidas no estado do Rio Grande do Sul apresentaram alta incidência destes patógenos. Quanto ao Bipolaris sp., esse fungo é comumente encontrado em áreas aonde se cultivam outros cereais de estação fria, como trigo e cevada, nas quais o patógeno encontra condições de sobrevivência. Conforme Neegard (1979), o fungo Bipolaris sp. possui efeito negativo na germinação das sementes e por conseqüência no estabelecimento de plântulas. Farias et al. (2005) concluiu que qualquer espécie deste patógeno causa redução na germinação em sementes de aveia preta. Resultado semelhante foi observado nesse estudo, pois as sementes com maior incidência de fungos foram as que apresentaram menor vigor. Segundo Neergard (1979), o tratamento com fungicidas é importante para controlar patógenos presentes nas sementes, conferir proteção nos casos de semeaduras profundas e possibilitar o escape do ataque de patógenos do gênero Fusarium, como F. nivale e $F$. culmorum, agentes causais do crestamento da aveia.

TABELA 2. Incidência dos fungos Fusarium spp. e Bipolaris sp. em sementes de aveia-preta, produzidas no município do Capão do Leão, safra 2005/06, tratadas com diferentes fungicidas.

\begin{tabular}{ccc}
\hline Tratamentos & Fusarium spp. $(\%)$ & Bipolaris sp. $(\%)$ \\
\hline Testemunha & $6 \mathrm{ab}$ & $3 \mathrm{a}$ \\
Carbendazin + Thiram & $0 \mathrm{~b}$ & $0 \mathrm{a}$ \\
Carboxin + Thiram & $0 \mathrm{~b}$ & $0 \mathrm{a}$ \\
Trichoderma spp. & $11 \mathrm{a}$ & $3 \mathrm{a}$ \\
\hline
\end{tabular}

Comparação de médias feita pelo teste de Duncan, a 5\% de probabilidade

Com relação à análise de isoenzimas, avaliando-se o perfil eletroforético das enzimas álcool desidrogenase, fosfatase ácida e esterase, observa-se um aumento na intensidade de bandas nos tratamentos nos quais foi utilizado o biopropetor e na testemunha sem tratamento. (Figura 1). Esse resultado pode ser atribuído a uma maior incidência de fungos observada nesses tratamentos, pois segundo LuccaFilho (1985), a ação bioquímica de microrganismos está relacionada com a ação de enzimas envolvidas na degradação da parede celular do tecido vegetal, a produção de toxinas as quais irão causar decomposição dos tecidos vivos, aumento da taxa respiratória do hospedeiro, atividade das enzimas da mitocôndria e interferência no metabolismo normal de aminoácidos. Segundo Delouche e Baskin (1973), a atividade enzimática pode indicar transformações degenerativas nas sementes. Em trabalho conduzido por Bock (1999), verificouse uma alta atividade da enzima álcool desidrogenase em sementes de soja, indicando aumento na respiração em função do acréscimo no grau de hidratação das sementes. $\mathrm{O}$ aumento na atividade celular ocorre a expensas das substâncias de reserva das sementes, que são transformadas em energia para a manutenção dos tecidos vivos das sementes e de novos compostos para o reparo de estruturas danificadas pela deterioração (Bewley e Black, 1994). 

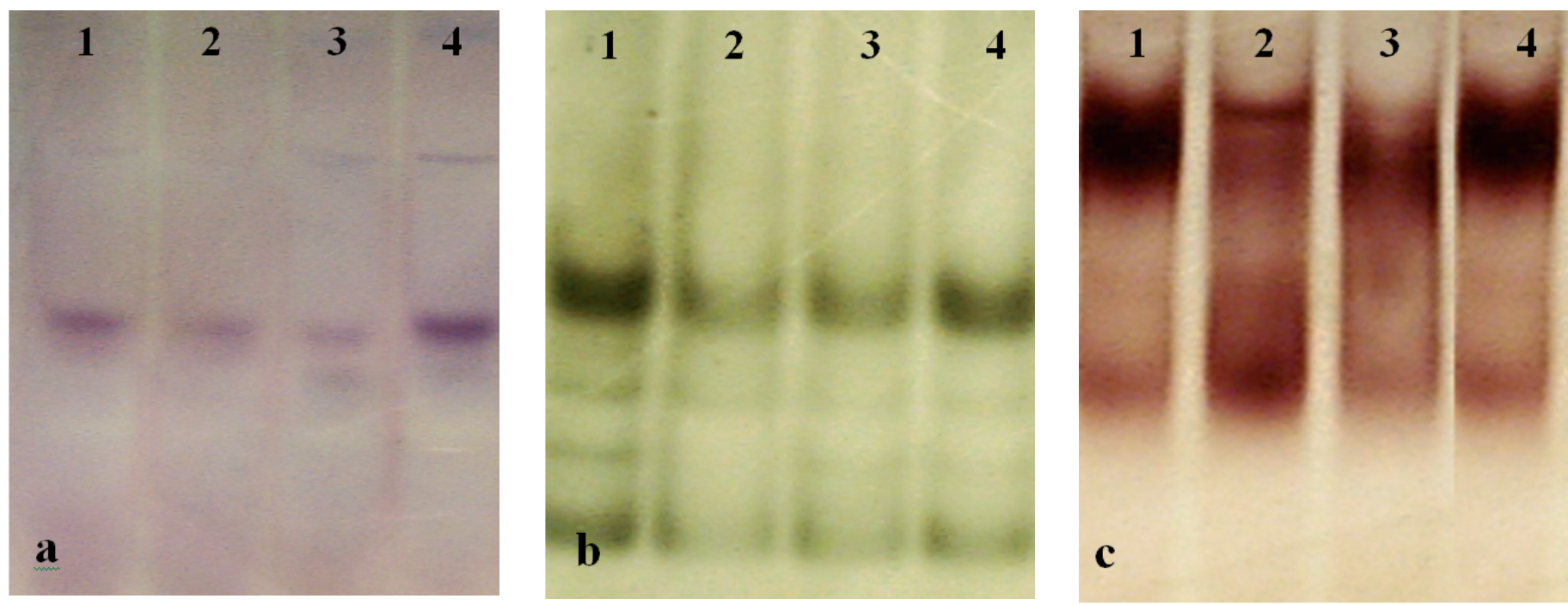

FIGURA 1. Padrões eletroforéticos das enzimas: a) Álcool Desidrogenase, b) Fosfatase Ácida e c) Esterase, em sementes de aveia-preta, produzidas no município do Capão do Leão na safra 2005/06, tratadas com os fungicidas: 1 - Testemunha, 2 - Carbendazin + Thiram, 3 - Carboxin + Thiram, 4 - Trichoderma spp.

A fosfatase ácida participa em reações de hidrólise de ésteres e pode provocar a peroxidação dos fosfolipídios de membrana. Esta enzima está envolvida também na manutenção do fosfato celular e sua atividade pode afetar o metabolismo do fosfato em sementes, como os níveis de ATP e nucleotídeos (Camargo et al. 2000). Conforme resultados apresentados nesse trabalho, a presença de fungos como Fusarium moliniforme e Bipolaris, pode ter contribuído para o aumento da atividade dessa enzima. Resultados semelhantes foram encontrados por Silva et al. (1998), que observou aumento na intensidade de bandas das enzimas fosfatase acida e esterase em de coleóptilos de milho infectados com Fusarium moliforme.

Quanto à enzima esterase, observou-se que assim como para as demais enzimas avaliadas, sementes com menor vigor apresentaram incremento na expressão dessa enzima. A esterase é uma enzima envolvida em reações de hidrólise de ésteres, estando diretamente ligada ao metabolismo dos lipídios, como os fosfolipídios totais de membrana. Segundo Roberts (1973), enzimas hidrolíticas têm sua atividade incrementada com a perda da viabilidade das sementes. Em estudos conduzidos por Santos et al. (2005), observou-se aumento na atividade da enzima esterase nas sementes de feijão durante o armazenamento. Estes mesmos pesquisadores verificaram nas sementes de feijão uma redução acentuada na germinação, primeira contagem de germinação, emergência em campo, pois alterações nos padrões desta enzima evidenciam a ocorrência de eventos deteriorativos.

Segundo Carvalho et al. (2000), os testes mais sensíveis para determinar o estádio de deterioração das sementes são aqueles que medem a atividade de determinadas enzimas associadas com a degradação das reservas e/ou biossíntese de novos tecidos, o mesmo foi confirmado no presente trabalho, pois as sementes que apresentaram redução na qualidade fisiológica devido ao ataque de patógenos, tiveram incremento na atividade enzimática.

\section{CONCLUSÕES}

O tratamento de sementes de aveia-preta com fungicidas químicos é uma alternativa eficiente no controle de patógenos em sementes.

Sementes tratadas com fungicidas químicos apresentam desempenho fisiológico e sanitário superiores em relação às tratadas com biopropetor e a testemunha sem fungicida.

Há maior atividade das isoenzimas álcool desidrogenase, fosfatase ácida e esterase em sementes com maior incidência de patógenos.

\section{REFERÊNCIAS}

ALFENAS, A.C. Eletroforese de isoenzimas e proteínas afins: funda,entos e aplicações em plantas e 
microrganismos. Viçosa: UFV, 1998. 574p.

BALARDIN, R.S.; LOCH, L.C. Efeito de thiram sobre a germinação de sementes de centeio e aveia. Revista Brasileira de Sementes, v.9, n.1, p.113-117, 1987.

BACKER, R. Improved Trichonomas spp.fot promoting crop productive. Trends in Biotechnology. v. 7, n.2. p34-8, 1989.

BELMONTE, P.B.A Qualidade física das sementes fiscalizadas de aveia-branca (Avena sativa L.) aveia preta (Avena strigosa Schreb), azevém-anual (Lolium multiflorum Lam.) e milheto (Pennisetum americanum L. Reeke) comercializadas na safra 2001/2002 no estado do Rio Grande do Sul. 2002. 25 f. Dissertação (Mestrado em Ciência e Tecnologia de Sementes) - Faculdade de Agronomia Eliseu Maciel, Universidade Federal de Pelotas, Pelotas.

BEVILAQUA, G.A.; PIEROBOM, C.R. Qualidade sanitária e fisiológica de sementes de aveia-preta (Avena strigosa Schreb) da zona sul do Rio Grande do Sul. Revista Brasileira de Sementes, v.17, n.1, p.19-22, 1995.

BEWLEY, J.D.; BLACK, M. Seeds: physiology of development and germination 2. ed. New York: Plenum Press, 445p. 1994.

BOCK, F.L. Resposta a nível molecular doenvelhecimento artificial, natural e pré-condicionamento de sementes de soja. 1999. 27f. Dissertação (Mestrado em Ciência e Tecnologia de Sementes) - Faculdade de Agronomia Eliseu Maciel, UniversidadeFederal de Pelotas, Pelotas.

BRASIL. Ministério da Agricultura e Reforma Agrária. Secretaria Nacional de Defesa Agropecuária. Departamento Nacional de Defesa Vegetal. Coordenação de Laboratório Vegetal. Regras para análise de sementes. 1992.365 p.

CAMARGO, M.L.P.; MORI, E.S.; MELLO, E.J.; ODA, S.; LIMA, G.P. Atividade enzimática em plântulas de Eucalyptus grandis provenientes de sementes envelhecidas artificialmente e manualmente. Ciência Rural, v.10, n.2, p.113-122, 2000.

CANTERI, M.G.; ALTHAUS, R.A.; VIRGENS FILHO, J. S.; GIGLIOTI, E. A.; GODOY, C. V. SASM - Agri: Sistema para análise e separação de médias em experimentos agrícolas pelos métodos Scoft - Knott, Tukey e Duncan. Revista Brasileira de Agrocomputação, v.1, n.2, p.18-24. 2001.

CARVALHO, M.L.M.; VIERIA, M.G.G.C.; PINHO, E.R.V. Técnicas moleculares em sementes. Biotecnologia Ciência e Desenvolvimento, v.3, n.17, p.44-47, 2000.
DELOUCHE, J.C.; BASKIN, C.C. Accelerated aging techniques for precidicting the relative storability of seeds lots. Seed Science and Technology, v.1, n.2, p.427-452, 1973.

FARIA, A.Y.K.; NETO, D.C.; ALBURQUERQUE, M.C. de F. Atividade Antagônica In vitro de Trichoderma Harzianum a patógenos de sementes de algodoeiro. Revista Tropical, v.6, n. 1, p 59-68, 2002

FARIAS, C.R.J.; LUCCA-FILHO, O.A.; PIEROBON, C.R.; DEL PONTE, E.M. Qualidade sanitária de sementes de aveia-preta (Avena strigosa Schreb.) produzidas no estado do Rio Grande do Sul, safra 1999/2000. Revista Brasileira de Sementes, v.24, n.1, p.1-4, 2002.

FARIAS, C.R.J.; DEL PONTE, E.M.; LUCCA FILHO, O.A.; PIEROBOM, C.R. Fungos causadores de helmintosporiose associados às sementes de aveia-preta (avena strigosa, schreb.). Revista Brasileira Agrociência, Pelotas, v.11, n.1, p.57-61, jan./mar. 2005.

MERTZ, L.M.; HENNING, F.A.; ZIMMER, P.D. Bioprotetores e fungicidas químicos no tratamento de sementes de soja. Revista Ciência Rural, v.39, n.1, p. 1318, 2009.

HENNING, A.A.; HENNING, F.A. Microbiolization of soybean seeds. In: XIX Seminário Pan Americano de Semillas, Assunção. Conferencias y Resumenes de Trabajos Presentados. Assunción: FELAS, 2004. p. 330.

KRZYZANOWSKI, F.C.; VIEIRA, R.D.; FRANÇA NETO, J.B. Vigor de sementes: conceitos e testes. Londrina: ABRATES, 1999. 218p.

LUCCA-FILHO, O.A. Importância da sanidade na produção de sementes de alta qualidade. Revista Brasileira de Sementes, v.7, n.1, p.113-124, 1985.

LUCCA-FILHO, O.A.; PORTO, M.D.M.; MAIA, M.S. Fungos em sementes de azevém-anual (Lolium multiflorum Lam). e seus efeitos no estabelecimento da pastagem. Revista Brasileira de Sementes, v.21, n.2, p.142-147, 1999.

MARTINS CORDER, M.P.; MELO, I.S. Antagonismo “ in vitro" de Trichoderma spp a Verticillium dahliac. Kleb. Scientia Agrícola, 55:1-7-1998

NEERGAARD, P. Seed Pathology. 2. ed. London, MacMillan Press, v.2, 1979.

ROBERTS, E.H. Loss of seed viability: chromosomal and genetic aspects. Seed Science and Technology, Zürich, v.1, n.3, p.515-527, 1973. 
SANTOS, C.M.R.; MENEZES, N.L.; VILLELA, F.A. Modificações fisiológicas e bioquímicas em sementes de feijão no armazenamento. Revista Brasileira de Sementes, v.27, n.1, p.104-114, 2005.

SCANDÁLIOS, J.G. Genetic control of multiple molecular forms of enzymes in plants: a rewiew. Biochemical Genetics, v.3, n.1, p.37-79, 1969.

SILVA, E.A.A.; VON PINHO, E.V.R.; VIEIRA, M.G.G.; CARVALHO, M.L. M.; MACHADO, J.C. Padrões eletroforéticos de isoenzimas em coleóptilos de milho em associação com microorganismos. Revista Brasileira de Sementes, v.20, n.2, p.176-181, 1998.

SILVA, E.A.A.; VON PINHO, E.V.R.; VIEIRA, M.G.G.; MACHADO, J.C. Alterações dos padrões de isoenzimas em sementes de milho infectadas por fungos. Pesquisa Agropecuária Brasileira, v.35, n.9, p.1725-1732, 2000.

WINDHAM, M.T.; ELAD, Y; BAKER, R.A. Amechanism for increased plant growth induced by Trichoderma spp. Phythology, v.76, p. 518-521, 1986 